Fachmann sondern auch den Laien entzuicken müssen. Sprechen wir es nur aus, dass gerade in diesen lebensfrischen Bildern der Hauptreiz liegt, welcher derartigen Prachtwerken Eingang beim grösseren Publikum verschaffte, wie dies namentlich die von Gould herausgegebenen beweisen. Während aber die Gould'schen Werke durch ihren hohen Preis (eine Lieferung 3 Guineen) sich fast ausschliessend englischen Verhältnissen anpassen, nimmt das Sharpe'sche Unternehmen in erfreulicher Weise eine vermittelnde Stellung ein. Der Preis einer Lieferung von 8 Tafeln mit dem zugehörigen Text beträgt nämlich nur $12^{1 / 2}$ Shilling (4 Thaler 5 Sgr. Cour.) und erleichtert also die Anschaffung wesentlich, um so mehr als dieselben regelmässig vierteljährlich erscheinen. Dabei ist das Format, Gross-Quarto, dem unpractischen durch Gould eingeführten Imperial-Folio, welches die Benutzung so sehr erschwert, bei Weitem vorzuziehen.

Nicht unerwähnt dürfen wir lassen, dass der bekannte Anatom Dr. Murie die Anatomie und Osteologie der Eisvögel am Schlusse in einem besonderen Kapitel zu behandeln versprochen hat.

Nach dem Prospectus wird der Preis des Werkes, welches in einer Auflage von nur 200 Exemplaren erscheint, nach der Vollendung, die mit der 13-14 Lieferung erfolgen soll, ansehnlich erhöht. Ein Grund mehr mit der Theilnahme nicht zu zögern.

Wir schliessen daher mit dem aufrichtigen Wunsche, dass diese Zeilen dazu beitragen mögen, dem schönen und verdienstlichen Unternehmen, welches in England sich schon so viele Freunde erworben, auch in Deutschland in weiteren Kreisen Eingang zu verschaffen. Es bietet dem Fachmanne jede gewünschte Auskunft und verspricht dem Liebhaber und Freunde, als gelungenes Bilderwerk, manche genussvolle Stunde.

Bremen, im März 1870.

Ph. L. Martin, Die Praxis der Naturgeschichte. 3 Theile. Weimar 1869, Verlag von B. Fr. Voigt.

Unsere naturwissenschaftliche Literatur ist nicht arm an Werken, welche die praktische Seite der Naturgeschichte zum Gegenstand haben und welche wenigstens von Anfängern als Lehrbiicher mit Vortheil zu gebrauchen sind. Wenn also auch nicht Bedürfniss nach Vermehrung dieser literarischen Hülfsmittel vor- 
handen, so begrüssen wir doch mit Freude das Erscheinen eines neuen Werkes in diesem Gebiet, da es erschöpfender als alle übrigen nach allen Richtungen den umfangreichen Stoff behandelt und in sich zusammenfasst, was jene ergainzungsweise uns bieten, wodurch auch dem Fachmann ein nuitzliches Handbuch geboten wird. Eingedenk dem Vorsatz, etwas vollständiges zu liefern, beschränkt sich auch der Verfasser nicht nur auf eine Anleitung zum Sammeln und Präpariren todter Naturkörper, sondern verspricht, in dem dritten Theile die Behandlung lebender Thiere, deren Erhaltung und Pflege in der Gefangenschaft sowie im freien Zustande abzuhandeln.

Der erste Theil von Martin's Praxis der Naturgeschichte, welcher uns vorliegt, enthält die Taxidermie. Von den vier Ąbschnitten, in welchen das hierauf Beziigliche besprochen wird, betrifft der erste die Konservirmittel. Der Verfasser führt uns die zerstörenden Einflüsse vor, denen die Organismen nach dem Tode ausgesetzt sind, erläutert ausführlich die Bedingungen der Fäulniss und leitet davon die Mittel her, die jenen Einflïssen Widerstand leisten und einen Körper, wenigstens auf lange Zeit, vor dem Untergang schützen können. Es muss besonders rühmend hervorgehoben werden, dass der Verfasser nicht nur, wie in vielen ähnlichen Werken es geschehen, Recepte aufführt und diese, ohne ihre Wirkungsweise näher zu erklären, als ein für alle Male gültig hinstellt, sondern die Wirkungen der verschiedensten Mittel untersucht und, ohne sein subjectives Urtheil zu verhehlen, mit allen Arten der Konservirung bekannt macht, welche angewendet wurden und noch im Gebrauche sind; es findet sogar das Balsamiren der alten Egypter genaue Besprechung.

Das Naturaliensammeln und Präpariren auf Reisen nimmt das zweite Kapitel ein. Durch einen jahrelangen Umgang mit Naturalien mit der häufigen Unzulänglichkeit in der Präparation und den daraus für den Conservator erwachsenden Widerwärtigkeiten bekannt, hat der Verfasser diesem Theil eine besondere Sorgfalt gewidmet. Bei den aufgestellten Principien haben Ihn hauptsächlich seine eigenen, im tropischen Amerika gemachten Erfahrungen geleitet und wir finden daher nicht eitle, am Schreibtisch ausgeheckte Ideen, sondern praktisch geprüfte und bewährte Vorschriften. Zum Messen der Vögel möchten wir noch einiges hinzufügen. Es werden zwei Maasse vorgeschrieben, welche der reisende Sammler von frischen Exemplaren nehmen soll, nämlich 
die Länge der Schnabelspitze bis zum Schwanzende und die Differenz zwischen den Flügelspitzen und diesem. Wenn auch für das wissensehaftliche Bestimmen eines Vogels diese beiden Maasse vorläufig vollständig genügen, da alle übrigen, welche in dieser Hinsicht Wichtigkeit haben, später von dem Balge genommen werden können, so müssen doch für den Conservator noch die Verhältnisse des Cadavers aufgezeichnet werden, da solche zur richtigen Darstellung der Figur des Vogels durchaus nothwendig sind. Es sind diese Maasse: die Länge des Halses, die Länge des Rumpfes und die Breite desselben über die Schultern. Die Fixirung von Thierfährten, zu welcher am Schlusse dieses Kapitels aufgemuntert wird, hat gewiss für ein richtiges Aufstellen der Thiere grosse Wichtigkeit, kann aber von dem reisenden Forscher doch nur in beschränkter Weise durchgeführt werden.

In dem Ausstopfen der Thiere, das im dritten Abschnitt besprochen wird, bekennt sich der Verfasser zur NaumannschenSchule. Es ist diese Methode gegenwärtig die allgemeinste und geniigt auch vollständig. Dass die vom Verfasser begründete Dermoplastik, die im zweiten Theil dieses Werkes zur Abhandlung kommen soll, eine grosse Zukuntt haben und die gegenwärtige Art des Ausstopfens verdrängen wird, möchten wir noch abwarten.

Einige Bemerkungen über den Naturalienhandel, das Verpacken und Versenden von Naturalien, sowie eine Aufzählung: der grösseren derartigen Handlungen bilden das letzte Kapitel.

Schliesslich müssen wir noch der fünf beigegebenen Tafeln Erwähnung thun. Die von Herren Fr. Specht sehr gut ausgeführten Zeichnungen dienen theils zur Erläuterung des Textes, theils bilden sie in den gelungenen Wiedergaben von Säugethieren und Vögeln versehiedener Familien sehr brauchbare Vorlagen bei der Präparation solcher Bälge.

Ant. Reichenow.

\section{Etwas über die Acclimatisationsfähigkeit des Goldfasans,} (Thaumalea picta).

\section{Carl Baron Drachenfels.}

Doctor A. Brehm spricht in seinem "Thierleben" an der betreffenden Stelle die Ansicht aus, dass der Goldfasan im Süden 


\section{$2 \mathrm{BHL}$ Biodiversity Heritage Library}

1870. "Ph. L. Martin, Die Praxis der Naturgeschichte." Journal fu

r Ornithologie 18, 380-382. https://doi.org/10.1007/bf02252491.

View This Item Online: https://www.biodiversitylibrary.org/item/103205

DOI: https://doi.org/10.1007/bf02252491

Permalink: https://www.biodiversitylibrary.org/partpdf/141860

\section{Holding Institution}

Smithsonian Libraries

\section{Sponsored by}

Biodiversity Heritage Library

\section{Copyright \& Reuse}

Copyright Status: Public domain. The BHL considers that this work is no longer under copyright protection.

This document was created from content at the Biodiversity Heritage Library, the world's largest open access digital library for biodiversity literature and archives. Visit BHL at https://www.biodiversitylibrary.org. 